\section{ZÖPPRITZ ON OCEAN CURRENTS}

I stivin SEND you a translation by a friend of an important contribution to the theory of ocean currents by Prof. Zöppritz, of Giessen, which has recently appeared in the Annalen der Hydrographie und Maritimen Meteorologie. The mathematical part of the subject has been published in the Annalen der Physik for April last, a translation of which will be found in the Philosophical Magazine for September.

One of the main objections urged against the theory that ocean currents are due to the impulse of the winds is that the winds can, it is alleged, produce only a surface drift, whereas many of the currents extend to great depths. I have always maintained that this objection is totally erroneous; that if the surface of the ocean be impelled forward with a constant velocity by the wind or by any other cause whatever, the layer immediately below will be dragged along with a constant velocity somewhat less. The layer underneath this second layer will in turn be also dragged along with a velocity less than the one above it. The same will take place in regard to each successive layer, the velocity of each being somewhat less than the one immediately above it, and greater than the one below it. In this manner the surface velocity may be transmitted downwards to any depth. This conclusion bas now been demonstrated by Prof. Zöppritz, in the following paper, to be perfectly correct.

JAMES CROLL

Though for a long time the majority of seamen and geographers have firmly held the opinion that the great equatorial ocean currents derived their origin from the trade winds, yet, so far as I know, no attempt has yet been made to treat the physical problem of the propagation of surface-velocities downwards through a very thick stratum of water, with the means presented by the theory of the friction of fluids, as elaborated within the last thirty years. Such an attempt is all the more demanded as many authors have lately denied that surface-forces could set the sea in motion to any considerable depth. At the same time the most groundless assumptions have been set forth as to the depth of such drift-currents.

The essential principle of the theory of the internal friction of fluids is that when a plane stratum of water is moved forward, by any cause, in its own plane with a given velocity, the adjoining stratum cannot remain at rest, but, in consequence of its molecular cohesion experiences an impulse to move in the same direction. And if the velocity of the former stratum be continuous the latter assumes a velocity which tends to approximate constantly to the given velocity. This second stratum now exerts the same influence on a third adjoining stratum that it had to suffer from the first, and sets it in motion in the same direction. The third stratum draws with it in a similar manner a fourth, a fourth a fifth, and so on. The propagation of the velocity is only bounded by the limits of the fluid itself. If thes a limits consist of a solid plane parallel to the strata, then the propagation of the velocity will cease only at this point, i.e., between the last liquid stratum and the first solid stratum.

The law according to which two neighbouring strata of velocities mutually influence one another has already been demonstrated by Newton, and the accelerating force exerted by the friction has been assumed as independent of the pressure and proportional to the difference of velocity. The later theory of the friction of fluids carries out this fundamental hypothesis as to the propagation of velocity between strata of the same propagation of lie at an indefinitely small distance $\xi$ from one another, and have accordingly only an indefinitely small difference of velocity $\Delta$, inasmuch as it makes the acceleration produced by the friction, at the plane in which the strata meet, proportional to the quotient $\Delta: \xi$ The factor $k$, by which this quotient must be multiplied in order to give the acceleration, is called the Coefficicut of Interial Friction.

The Newtonian hypothesis can be applied likewise to those parts of the bounding-surfaces of the fluid (where it is in contact with other bodies) which may possess independent motion. Here the acceleration produced by the limiting medium (which may be solid, fluid, or gaseous) is proportional to the difference of velocity which may in this case be finite. The factor of the proportion is called the Coefficient of External Friction. If the bounding body is a solid or even a fluid, then the fluid may wet it, that is, the stratum of fluid touching the limiting body may cling so fast to that body as to assume the same velocity. The coefficient of external friction is in this case infinitely great. This is the case between wood and water, glass and water; and, on the other hand, not so between glass and quicksilver.

The theory founded on this simple hypothesis has been subjected to the most varied experimental tests, and has, on the whole, been found to agree with the facts, so that the hypothesis may be regarded as proved.

In order to apply this theory to ocean currents, the simplifying presupposition has been made that the ocean is a mass of fluid contained between two horizontal planes at the distance $h$ from one another, but in other respects unbounded. On the surface of this mass of fluid a wind of uniform strength and direction is acting at all times, while the under-surface wets a solid plane, the seabottoin, and is therefore always at rest.

We must not, however, look on the action of the moving air on the surface stratum of the water as proceeding according to the Newtonian hypothesis; it will act in this way only so long as the surface remains level.

But the wind produces waves and acts on them according to quite different laws. One fact of experience is available here, viz., that the surface-stratum of the ocean under the influence of a uniform wind, moves in the direction of the wind with a constant velocity dependent on the strength of the wind. If, therefore, we place on the velocity of the water at the surface the condition that it has a value $w_{0}$ at all times given, everywhere uniform, and of uniform direction, then the problem of the determination of the internal velocity becomes soluble.

But the simplifying presuppositions here assumed are almost realised in the central equatorial regions of the great ocean; the solution of the problem becomes, therefore, of deep interest.

The following are the chief results of the solution :-

If for an infinitely long time the surface-stratum has been kept at an unchanging velocity, then the whole mass of water is in a steady state of motion, i.e., a state which no longer varies according to the time. The velocity $w$ is then dependent only on the depth $x$ beneath the surface, and diminishes in proportion as the depth increases, till at the bottom it reaches zero. This relation is expressed by the formula

$$
w=w_{\mathrm{c}} \frac{h-x}{h} .
$$

Naturally it is presupposed that no other causes, e.g., displacing currents, affect the motion of the deeper strata. If these deeper strata are kept by any foreign cause whatsoever in steady motion in a direction exactly opposite to the assumed motion, then at some point between the highest and the deepest strata there lies a plane where the velocity $=0$. If this plane lies at the depth $h_{1}$, then in the mass which lies above it the velocity follows the formula

$$
w=w_{0} \frac{h_{1}-x}{h_{1}},
$$

and is therefore in the same condition as if the strata that lie beneath were a solid mass.

It is specially noteworthy that the velocity is independent of the coefficient of friction, i.e., that in the 
state of motion that prevails after an infinitely long time the distribution of the velocity is the same in a thin fluid like water and in a thick fluid like syrup. In the fixed state of motion the infuence of friction is shown by the participation of all the strata in the motion which is imparted from without to the surface alone. Dependence on the coefficient of friction takes place only on the consideration of motions that vary with the time, and affords a measure for the depth of penetration of a surfaceimpulse within a given time.

The formula which gives the velocity at the depth $x$ of a mass of water originally at rest when for the time $t$ the surface has been kept at a constant velocity $w_{0}$, has naturally a less simple form than the formula which was found for steady motions. (The formula is the same as that which determines the propagation of heat in a solid wall whose one side is kept at a temperature $w_{0}^{\circ}$, whilst the other remains at $0^{\circ}$.) From this formula results the simple law that any velocity whatever between $o$ and $w$ o prevails at different times at depths which are related to one another as the square roots of the times. I have used the formula to compute the time that a point at the depth of 100 metres requires to attain half the surface velocity, i.e., $\frac{1}{2} w_{0}$. The coefficient of friction of the water was assumed according to $\mathrm{O}$. E. Meyer's determination, at 0 oI 44 , in which centimetres and seconds are the units of calculation. The result was that 239 years are required for the layer of water 100 metres deep to assume the half of the surface velocity. If it be asked what length of time is required for one-tenth of the surface velocity to penetrate to that depth, the answer is 4I years. Accordingly, the same velocities will be attained at a depth of Io metres after 2.39 and 0.41 years respectively. In a more viscous fluid the resulting numbers would be smaller.

These numbers are well calculated to give an idea of the slow rate at which changes of motion are propagated downwards. For the numbers computed for the propagation of a given surface motion, hold likewise for the penetration of a change of the motion from the surface downwards, whose influence is simply added to the already existing motion. A steady current, therefore, whose velocity diminishes linearly according to the depth, will sustain only an extremely slight alteration (except in the strata nearest the surface) from passing changes of motion that affect the surface, e.g. from contrary winds or storms. There will prevail, rather, at every deeplying point of this current, a mean velocity that changes only very slightly according to the time, and which is determined by the mean velocity at the surface. This latter velocity has the direction of the prevailing wind, according to whose strength it varies by a law that cannot be more accurately settled.

If the surface velocity varies periodically according to the time, as is the case with all winds that depend on seasons and the hours of the day, then, after this periodic state has lasted an infinitely long time, the relocity at all depths is a periodic function of the time of similar period, but such that the amount of variation decreases rapidly according to the depth and that the occurrence of the maxima and minima is delayed proportionally to the depth. At a depth of ro metres the amount of the yearly oscillation is already diminished to less than $\frac{1}{13}$ th ; at a depth of 100 metres it is beyond observation; at this depth the velocity is that corresponding to the steady state when the mean annual velocity is given to the surface. When the depths decrease in arithmetical proportion, the amounts of the oscillation decrease in geometrical proportion such that at four depths $x_{1}, x_{2}, x_{3}, x_{4}$, which stand in the relation

$$
x_{4}-x_{3}=x_{2}-x_{1} \text {; }
$$

the amounts $D_{1}, D_{2}, D_{3}, D_{4}$ stand in relation

$$
D_{4}: D_{3}=D_{2}: D_{1} \text {. }
$$

A maximum and the following minimum of the annual oscillation aiways exist at the same time at a vertical distance of II $^{\circ} 9$ metres.

To give a conception of the time that a constant surface-velocity which begins at the time $t=0$ requires, in order to bring the interior of an ocean 4,000 metres deep, which was previously at rest, to the state of steady motion, the following numbers will serve :-After 10,000 years there prevails at the half-depth, i.e., at $x=2,0 \times 0$ metres, just the velocity $0^{\circ} 037 w_{0}$. Since, according to the already-stated formula, in the steady state the velocity $0.5 w_{0}$ must prevail at this point, it is easily seen how far the ocean is still removed after 10,000 years from the steady state. After 100,000 years the velocity at the depth stated is already $0.461 w_{0}$, therefore very near the definitive value. After 200,000 years it differs only by two units in the third decimal place.

Among the results we have found, particular emphasis is to be laid on two, which seem more or less to contradict the views which have prevailed up to this time. In the first place, the steady motion arising in the interior of an unlimited stratum of water from an unvarying surface velocity makes itself felt with linearly decreasing velocity down to the bottom. Hitherto the view frequently expressed was, that the influence of surface currents, e.g., the drift caused in the tropical ocean by the trade winds, reached only to very moderate depths. Secondiy, it was found that all variations according to time, whether periodic or aperiodic, of the forces acting on the surface, propagate themselves downwards with extraordinary slowness, the periodic in very quickly decreasing amount. Taking both statements together, it follows that the movement of the chief part of a stratum of water exposed to periodically varying surface forces is determined by the mean velocity of the surface, and that the periodic variations are observable only in a comparatively thin surface stratum. From this it is obvious that hitherto the influence of the friction was undervalued in one direction, in so far, namely, as it was believed that its influence need not be considered as penetrating so deep, but in another direction it was overvalued, as too great an influence was wont to be ascribed to friction in respect of the propagation of varying current motions Its effect was also very much overvalued in another point, viz., in respect of the action of a bank on a stream flowing along it. If, I repeat, the whole surface is kept at a constant velocity, then also in the current bounded at the side the distribution of velocity in the steady state is independent of the co-efficient of friction. Beyond that, the influence of the banks on the distribution of velocity is exceedingly slight.

A further result is that two steady currents flowing parallel to one another, but in opposite directions, in a fiuid-stratum of constant depth, may very well graze one another without mutual disturbance. Their surface of division is then a vertical plane parallel to their direction in which the velocity o prevails, and which, therefore, stands to each current in the relation of a solid bank.

We have already shown numerically how extraordinarily slow the velocity existing at the surface is propagated downwards when the interior was previously at rest. Hence it may be concluded, vice versâ, that when every point of the whole mass of fluid has at a given moment a given velocity varying according to the depth, and when from the same moment onwards the surface remains at rest, the effect of this initial state vanishes equally slowly, i.e., the ocean passes into the state of rest with the same slowness with which in the first case the surface-velocity was propagated into the interior. In fact the formulæ show that the times for the increase and decrease of the same fraction of the given velocity are expressed by the same number.

If from some cause or other strong currents had been generated in the ocean, say 10,000 years ago, these 
currents would certainly not have as yet disappeared, but would still be the chief agents in determining the movement of the ocean at great depths, supposing that the earth were completely covered by an ocean of the uniform depth of 4,000 metres.

The interruption by continents and islands of irregular form will contribute to weaken the effect of these former states of motion, not so much through the increased friction on the ocean-bed as through the reflex currents, which arise everywhere, crossing and impeding one another. But it must be observed after the above numerical proof of the extremely slow spread of local alterations of motion over the interior mass, that the difficulties of an exact computation must not be shirked, on account of the traditional expression: "Friction quickly uses up all these velocities."

It would be possible to determine by observations whether effects of former movements are still present in the ocean. There would be required for this purpose only comparative current-observations at the most varied depths, to be applied in the central parts of the great equatorial currents and of the region of calms. Yet, however, we dare not hope to be able to detect small remnants of interior motion with the same certainty with which the effect of the former high temperature of the earth, which disappears according to the same law, could be detected by subterranean observation of temperature, were one able to penetrate deep enough with the thermometer into the earth's crust.

The above computations give us also an idea how distant must be the time of the initial state. What a long time, for example, must we imagine the trade winds to have been blowing with their present extent and strength in order to be justified in assuming that the present state of motion of the equatorial currents is steady. For that about 100,000 years are needed, supposing we postulate a mean depth of 4,000 metres and do not take into account the deadening influence of continents and islands which must somewhat diminish that number. Every initial state, whatever it may have been, vanishes finally, and gives way to a steady state, only the time varies which is required to diminish the originally arising velocity to any required degree of smallness.

\section{OUR ASTRONOMICAL COLUMN}

The MElbourne Observatory.--The thirteenth official Report of the Board of Visitors of the Melbourne Observatory, with the annual statement of the Government Astronomer, is before us. Mr. Ellery reports that the new building to contain the magnetical and meteorological instruments registering continuously by photography is completed. The staff of the Observatory now consists of the director, with a chief assistant (Mr. White) and three junior assistants. The transit-circle is found to be inadequate for modern requirements, and the Board of Visitors lay stress upon the necessity of providing an instrument of greater pretensions, to enable Melbourne to co-operate effectively with European and American observers; the Sydney Observatory being already in possession of a very superior meridian-instrument, and one having been ordered, it is understood, for the observatory under the direction of Mr. Todd at Adelaide, it is hoped that a new transit-circle may soon be provided for Melbourne, and it is suggested that the necessary appropriation, about $1,200 l$., might be made in two annual votes, as two years will be required for the completion of the instrument.

The great reflector, though reported to be working satisfactorily, the mirrors retaining an excellent polish, and no marked signs of deterioration being visible, is occasionally subject to trifling derangements of its mechanism. Unfortunately the publication of the work with this instrument, the drawings of nebulæ, has been delayed by the loss of the gentleman who copied the drawings on stone. The drawings, however, now only require printing, and their publication is not likely to be long retarded. Mr. Ellery refers to the miscellaneous observations made during the year to which his report relates (to June 30,1877 ), including observations of D'Arrest's comet of short period, determination of positions of stars used by Mr. Gill during his expedition to Ascension, measures of southern double stars and of the polar and equatorial diameters of Mars, and of Saturn's ring. With regard to the use of the great reflector it is mentioned, "Out of 326 available nights I50 were unfitted for observation from unfavourable weather, bright moonlight interfered on 32, while 49 were occupied with visitors, which, together with about 20 nights during which the telescope was under repair, or which were unavailable from other causes, left only 75 nights upon which observations could be made." From the observations made during the year upon 77 of the smaller nebulæ in Sir John Herschel's "General Catalogue," it is gathered that while the actual aspect of many conforms precisely with Herschel's description, others are so considerably changed as to be only recognisable by their position. The only change detected in the great nebula about $\eta$ Argûs, since the drawing in March, 1875 , has been " a break or separation in one of the branches on the preceding side."

Observations of the satellites of Uranus were made on sixteen nights, and on the same number of nights the satellites of Mars (the announcement of tise discovery of which had been telegraphed to Mr. Ellery by Sir George Airy) were unsuccessfully sought for ; the failure to find these objects with certainty and ease Mr. Ellery considers "somewhat unaccountable," but the reader will hardly need to be reminded that there are other cases where the large reflectors have not proved so adequate for work as the large refractors: sooner or later, at Melbourne or elsewhere, we hope to see a large instrument of the latter class applied to the survey of the southern heavens : the real astronomical work in the northern hemisphere, the more precise micrometrical measures and more delicate observations falling to the task of the practical astronomer, have been, as yet, pre-eminently clue to the use of the refractor.

BIELA'S AND HALLEY'S COMETS.-There are near approximations between the orbits of these bodies not far from points which were first roughly indicated by Littröw, in a communication to the Vienna Academy in 1854, entitled "Balnnnähen zwischen den periodischen Gestirnen des Sonnensystemes." In heliocentric longitude $39^{\circ} 25^{\prime}$ (equinox of 1836 ) the distance between the two orbits is 0.032 (the earth's mean distance from the sun $=1$ ), and in $200^{\circ} 5^{\prime}$, the distance is as small as o'or I. At the former point the true anomaly of Halley's comet is $-94^{\circ} 9^{\prime}$, with the elements of 1836 , and that of Biela's $-7 \mathrm{I}^{\circ} \mathrm{I} \mathrm{7}^{\prime} ;$ at $200^{\circ} 5 \mathrm{I}^{\prime}$ the true anomaly of Halley's is $+104^{\circ} 59^{\prime}$, and of Biela's $+90^{\circ} 2^{\prime}$; we see then that on the last return of Halley's comet to these parts of space, though its orbit approached so near to that of Biela's, there was no near approximation of the two bodies. It will be remembered that Biela's comet also passes very near to the orbit of Tempel's comet I866 I., and consequently to the track of the November meteorstream.

\section{GEOGRAPHICAL NOTES}

AMONG the geographical notes in the January number of the new periodical issued by the Royal Geographical Society we find some interesting information regarding the work to be done by Mr. Keith Johnston's East African Expedition. He is instructed to gather data for constructing as complete a map as possible of the route, and to make all practicable observations in meteorology, 